\title{
Quantum superposition of a single microwave photon in two different 'colour' states
}

\author{
Eva Zakka-Bajjani ${ }^{1 \star}$, François Nguyen ${ }^{1 \star}$, Minhyea Lee ${ }^{2}$, Leila R. Vale ${ }^{1}$, Raymond W. Simmonds $^{1}$ \\ and José Aumentado ${ }^{1 \star}$
}

Fully controlled coherent coupling of arbitrary harmonic oscillators is an important tool for processing quantum information ${ }^{1}$. Coupling between quantum harmonic oscillators has previously been demonstrated in several physical systems using a two-level system as a mediating element ${ }^{2,3}$. Direct interaction at the quantum level has only recently been realized by means of resonant coupling between trapped ions $^{4,5}$. Here we implement a tunable direct coupling between the microwave harmonics of a superconducting resonator by means of parametric frequency conversion ${ }^{6,7}$. We accomplish this by coupling the mode currents of two harmonics through a superconducting quantum interference device (SQUID) and modulating its flux at the difference $(\sim 7 \mathrm{GHz})$ of the harmonic frequencies. We deterministically prepare a single-photon Fock state $^{8}$ and coherently manipulate it between multiple modes, effectively controlling it in a superposition of two different 'colours'. This parametric interaction can be described as a beamsplitter-like operation that couples different frequency modes. As such, it could be used to implement linear optical quantum computing protocols ${ }^{9,10}$ on-chip ${ }^{11}$.

The ability to create and manipulate quantum number states in a linear resonator is an important task in cavity quantum electrodynamics (QED; ref. 1). Early theory ${ }^{6,7}$ predicted that parametric frequency conversion could be a way to implement a tunable direct coupling between quantized modes of different energies. Classically, two harmonic oscillators coupled through a time-varying element, modulated at the difference of the resonator frequencies, will periodically exchange energy. At the quantum level, this can be used to swap the quantum states of two harmonic modes. In optics, the efficiency and quantum coherence of frequency up-conversion have been demonstrated using a pumped nonlinear crystal to couple light at different wavelengths ${ }^{12-14}$. However, in these experiments it is challenging to access the state dynamics because strong coupling rates are difficult to obtain ${ }^{15}$. In hybrid mechanical systems, strong parametric coupling, based on frequency conversion, has been recently achieved ${ }^{16,17}$, creating the possibility for the manipulation of quantum states of mesoscopic mechanical resonators ${ }^{18}$. In superconducting circuits, parametric processes have been used mainly to couple superconducting quantum bits (qubits) at their optimal points ${ }^{19}$, or to make quantum-limited microwave amplifiers ${ }^{20-23}$, yet little has been done with frequency conversion. Several circuit designs that enable frequency conversion between linear resonators have been proposed $^{21,24,25}$. This particular interaction can be combined with the powerful tools already available in circuit QED (ref. 8), where the creation and the detection of complex quantum states of the field have been demonstrated ${ }^{26}$. Here we measure the coherent dynamics of the parametric frequency conversion of a single photon between the first three internal resonant modes of a superconducting cavity, the state of which is prepared and read out with a superconducting qubit.

Our circuit consists of a quarter-wave $(\lambda / 4)$ coplanar waveguide (CPW) resonator terminated to ground via a SQUID (Fig. 1a). At the other end, it is coupled to a $50 \Omega$ transmission line through a capacitance $C_{\mathrm{c}}$ to weakly probe the resonator with microwave reflectometry. At this node we also couple it to a flux-biased phase qubit through an effective capacitance $C_{\mathrm{g}}$. This provides a single-photon source and detector for cavity photons. The SQUID is a superconducting loop asymmetrically intersected by three Josephson junctions. A 'pump' line allows both d.c. ( $\left.\Phi_{\mathrm{sq}}^{\text {d.c. }}\right)$ and microwave modulation $\left(\Phi_{\mathrm{sq}}^{\mu \mathrm{w}}(t)\right)$ of the global flux $\left(\Phi_{\mathrm{sq}}\right)$ in the SQUID loop. In the linear current regime (see Supplementary Information for an estimation of the current nonlinearities), and for frequencies lower than the plasma frequency, we can model the SQUID as a flux-dependent lumped-element inductor, $L_{\mathrm{sq}}\left(\Phi_{\mathrm{sq}}\right)$, modifying one of the boundary conditions of the cavity. Each cavity mode $n$ (assumed lossless in this model) behaves as a fluxtunable harmonic oscillator (Fig. 1b) with a resonance frequency $v_{\mathrm{n}}\left(\Phi_{\mathrm{sq}}^{\text {d.c. }}\right)=1 / 2 \pi \sqrt{C_{\mathrm{n}} L_{\mathrm{n}}^{\prime}\left(\Phi_{\mathrm{sq}}^{\text {d.c. }}\right)}$, where $L_{\mathrm{n}}^{\prime}\left(\Phi_{\mathrm{sq}}^{\text {d.c. }}\right)=L_{\mathrm{n}}+L_{\mathrm{sq}}\left(\Phi_{\mathrm{sq}}^{\text {d.c. }}\right)$ and $L_{\mathrm{n}} C_{\mathrm{n}}$ is the lumped series oscillator describing the modes of the bare cavity ${ }^{23,27,28}$. Figure 1c shows the spectroscopic data, measured as a function of both probe frequency and d.c. flux $\Phi_{s q}^{\text {d.c. }}$, for the first two modes, 0 and 1. The measurements shown in Figs 2 and 3 are performed at a flux bias point $\Phi_{\mathrm{sq}}^{\text {d.c. }}=-0.37 \Phi_{0}$ (point ' $\mathrm{A}$ ' in Fig. 1c), where $v_{0}=3.7 \mathrm{GHz}$ and $v_{1}=10.74 \mathrm{GHz}$ (to simplify the notation, the dependence on d.c. flux will not be specified for the cavity resonances). The $n=2$ mode cannot be directly probed in our setup, but is expected to be around $18 \mathrm{GHz}$. The extracted loaded quality factors are about 9,000 for both modes 0 and 1 . The SQUID inductance can be modulated by applying a small microwave flux $\Phi_{\mathrm{sq}}^{\mu \mathrm{w}}(t)=\delta \Phi_{\mathrm{sq}}^{\mu \mathrm{w}} \cos \left(2 \pi v_{\mathrm{p}} t+\varphi_{\mathrm{p}}\right)$, with $\delta \Phi_{\mathrm{sq}}^{\mu \mathrm{w}} \ll \Phi_{0}$ at a pump frequency $v_{\mathrm{p}}$. To first order in flux, the SQUID inductance is (the phase of the pump is defined modulo $\pi$, depending on the sign of the first derivative of the SQUID inductance with flux)

$$
\begin{gathered}
L_{\mathrm{sq}}\left(\Phi_{\mathrm{sq}}^{\text {d.c. }}+\Phi_{\mathrm{sq}}^{\mu \mathrm{w}}(t)\right)=L_{\mathrm{sq}}\left(\Phi_{\mathrm{sq}}^{\text {d.c. }}\right)+|\delta L| \cos \left(2 \pi v_{\mathrm{p}} t+\varphi_{\mathrm{p}}\right) \\
\text { with } \delta L=\delta L\left(\Phi_{\mathrm{sq}}^{\text {d.c. }}, \delta \Phi_{\mathrm{sq}}^{\mu \mathrm{w}}\right)=\left.\frac{\partial L_{\mathrm{sq}}}{\partial \Phi}\right|_{\Phi_{\mathrm{sq}}^{\text {d.c. }}} \delta \Phi_{\mathrm{sq}}^{\mu \mathrm{w}}
\end{gathered}
$$

When $v_{\mathrm{p}}=v_{\mathrm{m}}-v_{\mathrm{n}}$ (by convention $m>n$ ), frequency conversion is induced between modes $n$ and $m$ (refs 6,7,29). In a typical $\lambda / 4$ cavity, the mode frequencies are $v_{n}^{\lambda / 4}=(2 n+1) v_{0}^{\lambda / 4}$, and therefore a pump at $2 v_{0}^{\lambda / 4}$ can also couple other neighbouring modes by

${ }^{1}$ National Institute of Standards and Technology, 325 Broadway MS817.03, Boulder, Colorado 80305, USA, ${ }^{2}$ Department of Physics, University of Colorado, Boulder, Colorado 80309, USA. *e-mail: eva.zakka-bajjani@nist.gov; francois.nguyen@nist.gov; jose.aumentado@nist.gov. 


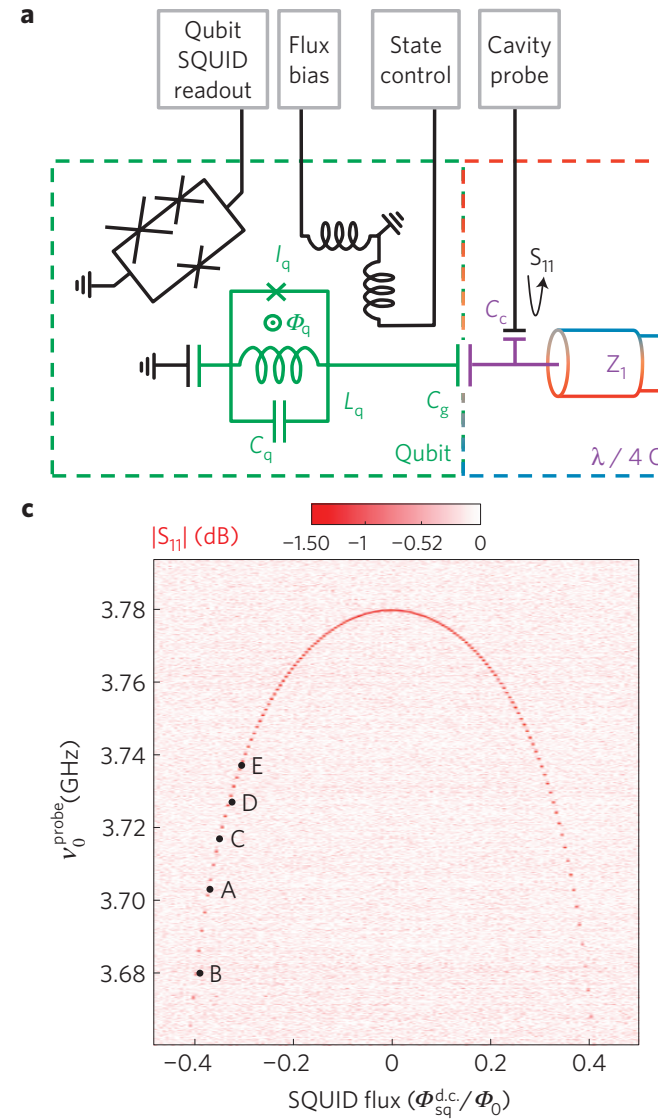

b
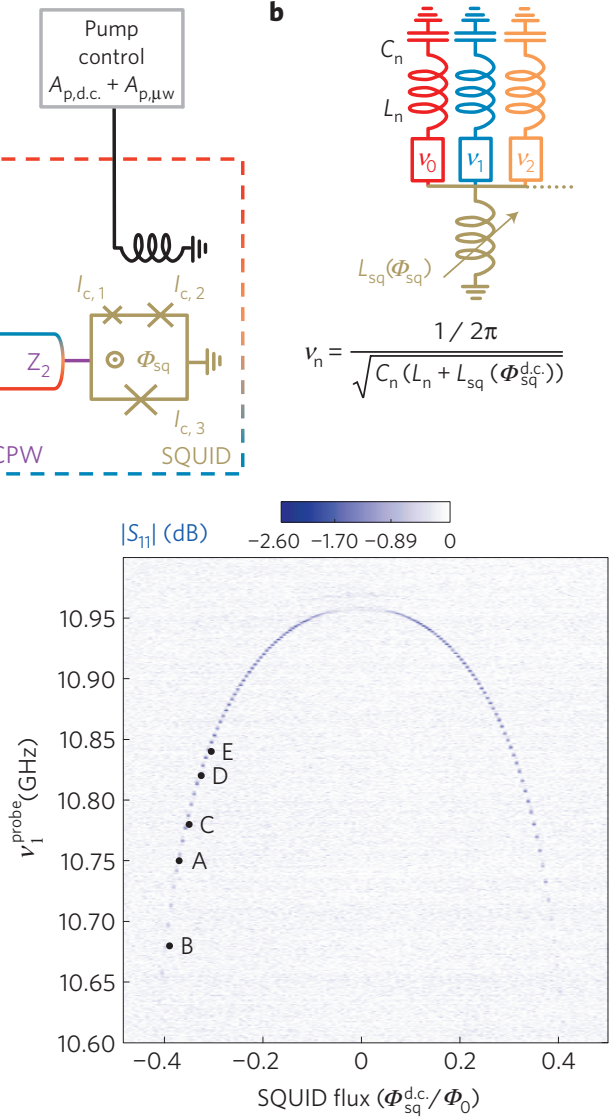

Figure 1 | Device description and spectroscopy. a, The 'Superconducting Parametric Beam Splitter' (SPBS) is a $\lambda / 4$ CPW resonator with a boundary condition that is varied by a SQUID. The cavity has a total length of $7.57 \mathrm{~mm}$ and is composed of two $\lambda / 8$ sections with characteristic impedances $Z_{1}=50 \Omega$ and $Z_{2}=46 \Omega$. The SQUID is intersected by three junctions of critical currents $I_{c, 1} \approx 0.21 \mu \mathrm{A}, I_{c, 2} \approx 0.41 \mu \mathrm{A}$, and $I_{c, 3} \approx 0.88 \mu \mathrm{A}$. The flux through the SQUID is d.c.-biased and microwave-modulated via an inductively coupled pump line. To allow reflectometry measurements, the SPBS is coupled to a $50 \Omega$ transmission line through a small capacitance $C_{c} \approx 1.8 \mathrm{fF}$. A phase qubit is capacitively coupled to the SPBS with a strength $g_{\mathrm{q} 1}=32 \mathrm{MHz}$

(Supplementary Information). We manipulate the qubit state with microwave pulses and tune its frequency with a flux bias line (a picture of the device is provided in Supplementary Information). Measurements are performed in a dilution refrigerator operated at $35 \mathrm{mK}$, which makes thermal noise negligible at our working frequencies. b, SPBS simple model. Each cavity mode $n$ is described by the equivalent $L_{n} C_{n}$ of the $\lambda / 4$ CPW nth harmonic in series with the SQUID tunable inductor. c, Measured reflection coefficient $\left|S_{11}\right|$ on $C_{c}$ as a function of both d.c.-flux in the SQUID and probe frequency.

conversion, as well as induce degenerate parametric amplification of mode 0 (ref. 29). To couple only two selected modes by frequency conversion (for a given pump frequency), we modify the cavity dispersion relation by slightly varying the characteristic impedance along the CPW resonator. This ensures that the frequencies $v_{n}$ are not equally spaced, satisfying the conditions: $\left|v_{2}-v_{1}\right|-\mid v_{1}-$ $v_{0}|,| v_{1}-v_{0} \mid-2 v_{0} \gg \Delta v_{0}, \Delta v_{1}, \Delta v_{2}$, where $\Delta v_{n}$ is the bandwidth of mode $n$ (we measure $\Delta v_{0,1} \lesssim 2 \mathrm{MHz}$ ). This allows us to restrict our description to a two-mode manifold. Following the usual treatment for two parametrically coupled oscillators ${ }^{29}$, one can write the classical equations of motion of the two normalized normal mode amplitudes $a_{\mathrm{k}}=\sqrt{L_{\mathrm{k}}^{\prime} / h \nu_{\mathrm{k}}}\left(\dot{I}_{\mathrm{k}} /\left(2 i \pi v_{\mathrm{k}}\right)+I_{\mathrm{k}}\right)$, where $I_{\mathrm{k}}$ is the current in mode $k(k \in\{\mathrm{n}, \mathrm{m}\})$, coupled by a quasi-resonant pump of frequency $v_{\mathrm{p}}=v_{\mathrm{m}}-v_{\mathrm{n}}+\Delta v_{\mathrm{p}}\left(\Delta v_{\mathrm{p}} \ll v_{\mathrm{m}}, v_{\mathrm{n}}\right)$. Keeping the resonant terms for each mode and invoking the rotating wave approximation, one has

$$
\begin{gathered}
\dot{a}_{\mathrm{n}}(t) / 2 \pi=-i v_{\mathrm{n}} a_{\mathrm{n}}(t)-i g_{\mathrm{mn}} \mathrm{e}^{i\left(2 \pi v_{\mathrm{p}} t+\varphi_{\mathrm{p}}\right)} a_{\mathrm{m}}(t) \\
\dot{a}_{\mathrm{m}}(t) / 2 \pi=-i v_{\mathrm{m}} a_{\mathrm{m}}(t)-i g_{\mathrm{mn}} \mathrm{e}^{-i\left(2 \pi v_{\mathrm{p}} t+\varphi_{\mathrm{p}}\right)} a_{\mathrm{n}}(t)
\end{gathered}
$$

where $g_{\mathrm{mn}}=1 / 2 \sqrt{v_{\mathrm{m}} \nu_{\mathrm{n}}}|\delta L| / \sqrt{L_{\mathrm{m}}^{\prime} L_{\mathrm{n}}^{\prime}}$ is the parametric coupling frequency. For simplicity, we neglect its dependence on the pump detuning. The quantum model is derived by identifying equation (2) with Heisenberg's equations of motion for the annihilation operators $\hat{a}_{\mathrm{n}, \mathrm{m}}(t)$ (the pump field is assumed to be strong enough to be described classically). The state associated with each harmonic mode of the cavity can be described in terms of quantized microwave excitations, namely photons. In the doubly rotating frame defined by the unitary transformation $\mathrm{e}^{2 i \pi\left[\left(v_{\mathrm{n}}-\Delta v_{\mathrm{p}} / 2\right) \hat{a}_{\mathrm{n}}^{\dagger} \hat{a}_{\mathrm{n}}+\left(v_{\mathrm{m}}+\Delta v_{\mathrm{p}} / 2\right) \hat{a}_{\mathrm{m}}^{\dagger} \hat{a}_{\mathrm{m}}\right] t}$, the Hamiltonian, in the Schrödinger representation, is

$$
\hat{H}_{I}=h \frac{\Delta v_{\mathrm{p}}}{2}\left(\hat{a}_{\mathrm{n}}^{\dagger} \hat{a}_{\mathrm{n}}-\hat{a}_{\mathrm{m}}^{\dagger} \hat{a}_{\mathrm{m}}\right)+h g_{\mathrm{mn}}\left(\hat{a}_{\mathrm{n}}^{\dagger} \hat{a}_{\mathrm{m}} \mathrm{e}^{i \varphi_{\mathrm{p}}}+\hat{a}_{\mathrm{n}} \hat{a}_{\mathrm{m}}^{\dagger} \mathrm{e}^{-i \varphi_{\mathrm{p}}}\right)
$$

When $\Delta v_{\mathrm{p}}=0, \hat{H}_{I}$ describes a generalized beam-splitter operation between modes of different frequencies that preserves the total number of photons ${ }^{1}$. The effective transparency is modulated by the parametric interaction duration $\Delta t_{\mathrm{p}}$. In particular, the complete swap of a given initial state from one mode to the other is achieved for a pump $\pi$-pulse of duration $\Delta t_{\mathrm{p}}=1 /\left(4 g_{\mathrm{mn}}\right)$.

Figure $2 \mathrm{a}$ and $\mathrm{b}$ show two separate sets of spectroscopic data probing the parametric coupling between modes $n=0$ and $m=1$. They are plotted as a function of $v_{\mathrm{p}}$ and driven at three different pump amplitudes $A_{\mathrm{p}, \mu \mathrm{w}}$ (measured at the output of the microwave generator). The probe frequency $v_{0,1}^{\text {probe }}$ is swept through $v_{0}$ (Fig. 2a), and $v_{1}$ (Fig. 2b). These data show well-resolved normal-mode 

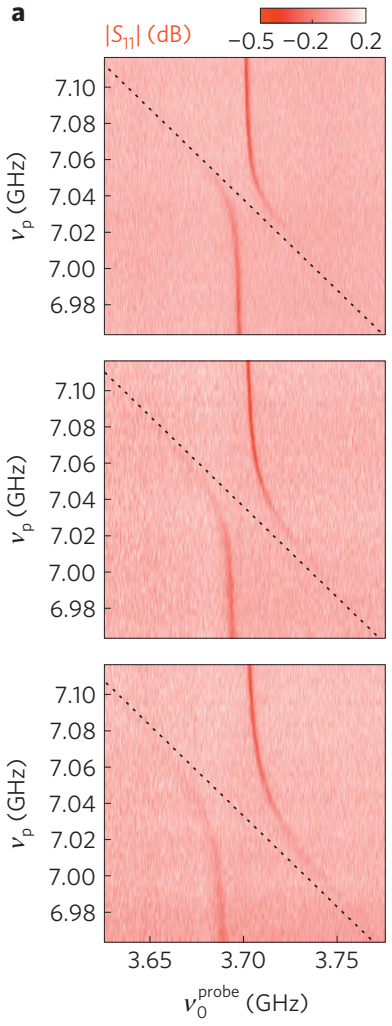

c

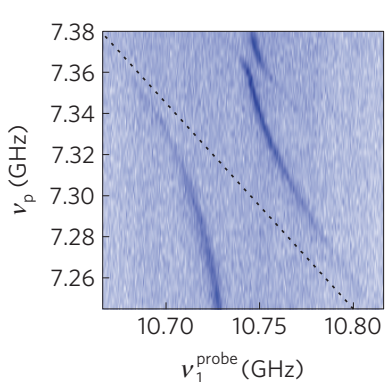

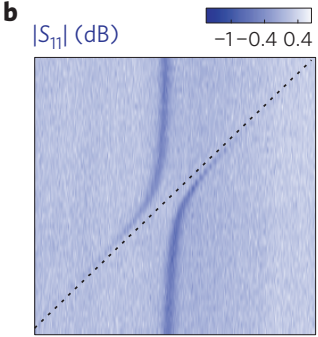
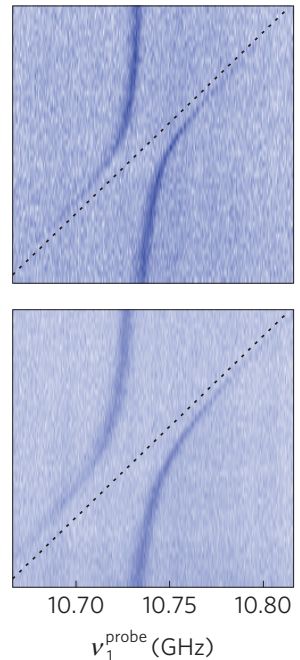

。
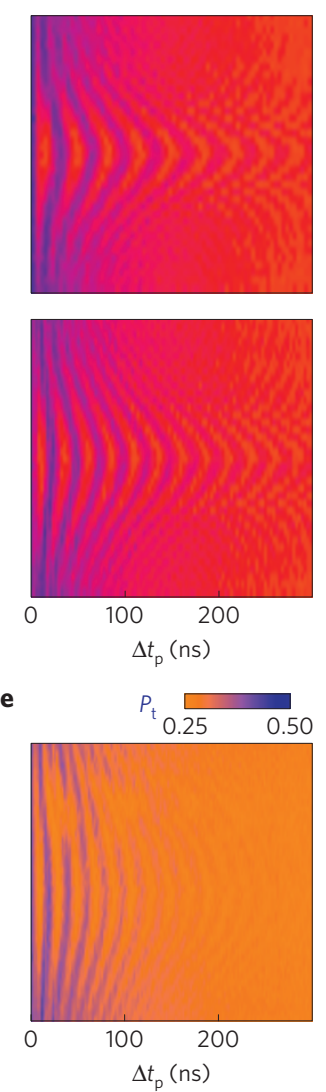
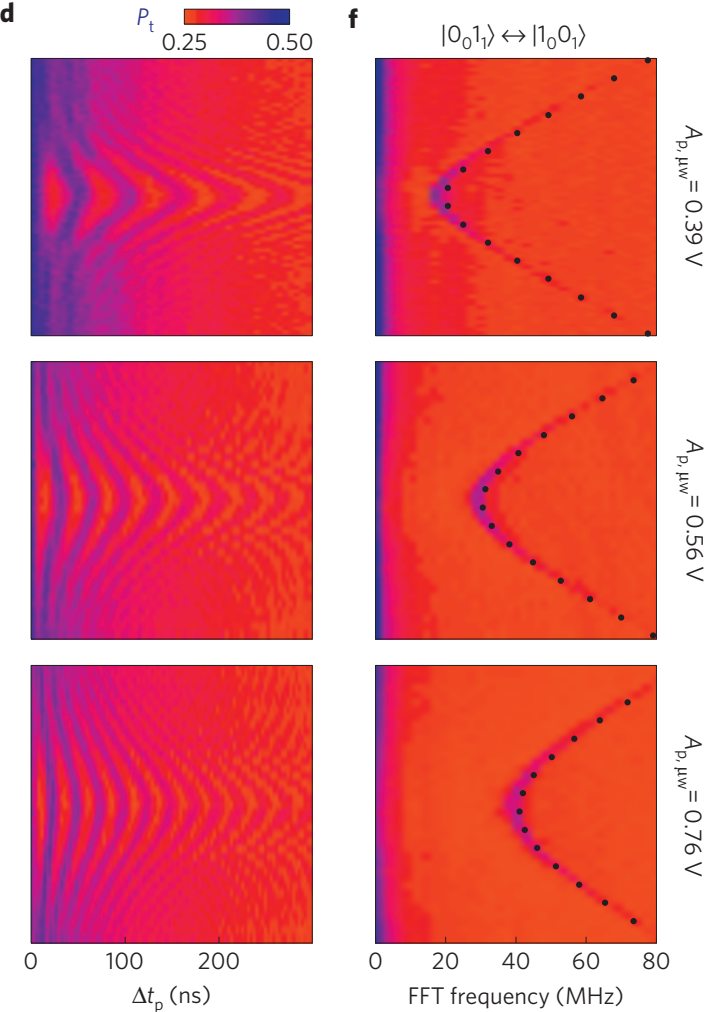

$\mathbf{g}$

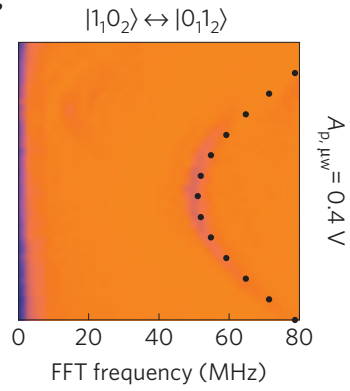

Figure 2 | Spectroscopy of parametrically coupled cavity modes and one-photon Rabi-swap oscillations. a-c, Spectroscopic data of modes 0 and 1 , in the presence of a microwave pump drive on the cavity SQUID, at $\Phi_{\mathrm{s}}^{\text {d.c. }}=-0.37 \Phi_{0}$ (point 'A' on Fig. 1c). $\left|S_{11}\right|$ is plotted in colour scale as a function of both probe and pump frequencies, when modes 0 and $1(\mathbf{a}, \mathbf{b})$, or 1 and $2(\mathbf{c})$, are distinctly coupled. Parametric interaction enables a strong coupling between harmonics that are $7 \mathrm{GHz}$ detuned, resulting in an avoided crossing between the two eigenmodes of the system (normal-mode splitting). The dashed black lines outline the asymptotic behaviour. The coupling rate $g_{10}$ depends linearly on the pump amplitude $A_{p, \mu w}$. For the same pump amplitude, the coupling rate $g_{21}$ between modes 1 and 2 is about $\sqrt{5} g_{10}$. We notice the presence of an unexplained parametrically coupled resonance at $v_{\mathrm{p}}=7.35 \mathrm{GHz}$.

d,e, Single-photon conversion oscillations. The colour scale encodes the tunnelling probability $P_{\mathrm{t}}$ of the qubit state as a function of both pump pulse duration and frequency. When the qubit is in the ground state, $P_{\mathrm{t}}$ is set to $\approx 25 \pm 2 \%$. This probability increases linearly with the excited state occupancy. When the qubit is prepared in the excited state, $P_{\mathrm{t}}$ is $\approx 50 \pm 2 \%$ (the amplitude of the colour scale is chosen as the readout contrast of the qubit). The amplitude of the conversion oscillations is about 15\% (see Supplementary Information). $\mathbf{f}$, , Fourier transform of the time-domain measurements showing the hyperbolic dependence between the coupling rate and the pump detuning. The theoretical curve is superposed with a dotted line.

splittings centred at $v_{\mathrm{p}}=v_{1}-v_{0} \approx 7.043 \mathrm{GHz}$, characteristic of the strong-coupling regime. In other words, the parametric coupling rate $g_{10}$ is larger than the bandwidths of the modes. The extracted $g_{10}$ rate is linearly dependent on pump amplitude, yielding up to $20 \mathrm{MHz}$ coupling for a flux modulation $\delta \Phi_{\mathrm{sq}}^{\mu \mathrm{w}} / \Phi_{0} \sim 2 \%$. As the SQUID inductance is slightly rectified by the pump modulation, we observe a small shift of the resonance frequencies, leading to a shift of the splitting centres (Supplementary Information). The 'quasi-resonant pump' condition, $v_{\mathrm{p}}=v_{1}-v_{0}^{\text {probe }}\left(v_{\mathrm{p}}=v_{0}+v_{1}^{\text {probe }}\right)$, describes the asymptotic behaviour at small pump detuning. We can also couple modes 1 and 2 (Fig. 2c). As we designed the resonator to shift the harmonic mode frequencies, this requires a pump frequency $\sim 250 \mathrm{MHz}$ higher than that needed for the $0 \leftrightarrow 1$ conversion. In addition, the $1 \leftrightarrow 2$ conversion rate is consistent with expectations from our simple model (equation (2)), $g_{21} \sim \sqrt{5} g_{10}$. As $g_{10}, g_{21}$ are small enough compared with $\left|v_{2}-v_{1}\right|-\left|v_{1}-v_{0}\right|$, we can, in fact, address these dual-mode manifolds separately.

As an illustration of the circuit efficiency at the quantum level, we realize the conversion oscillations of a one-photon Fock state between modes 0 and 1 ( 1 and 2 ). We prepare and measure the state of mode 1 using a superconducting phase qubit, following the method outlined in refs $26,30,31$. The qubit is well described by a two-level system, with a ground state $|\mathrm{g}\rangle$ and an excited state $|\mathrm{e}\rangle$. Their energy difference defines the qubit frequency $v_{\mathrm{q}}$, which we can tune from 7 to $12 \mathrm{GHz}$ by means of an external flux $\Phi_{\mathrm{q}}$ (Supplementary Information). The qubit state is manipulated using 


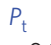

0.25

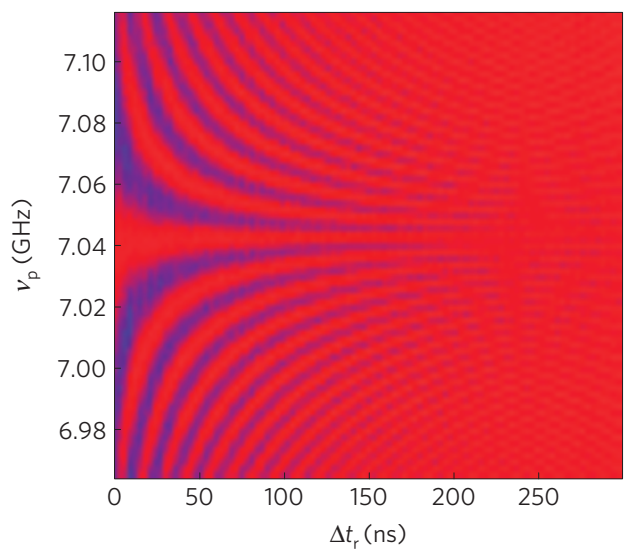

b

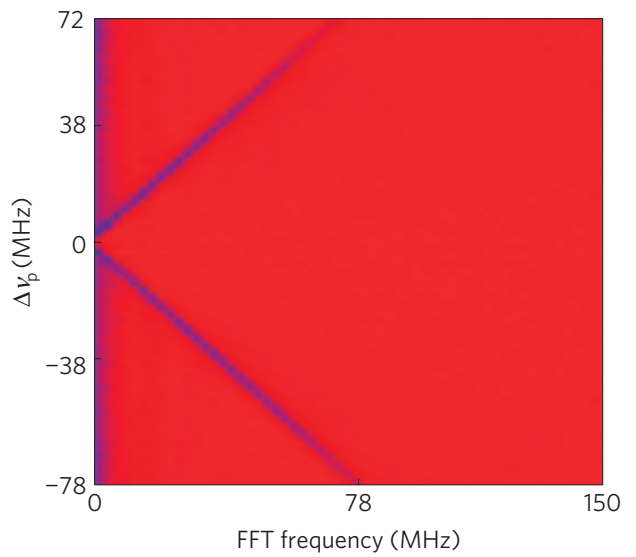

Figure 3 | Single-photon Ramsey interferences. a, Ramsey-fringes experiment with a single photon split between modes 0 and 1 , at $\Phi_{\mathrm{sq}}^{\text {d.c. }}=-0.37 \Phi_{0}$ and $A_{p, \mu w}=0.39 \mathrm{~V}$ at the microwave generator output. The cavity is initially prepared in the state $\left|0_{0} 1_{1}\right\rangle$. Two phase-coherent ' $\pi / 2^{\prime}$ pump pulses, separated by a variable delay $\Delta t_{r}$ are then applied. The state of mode 1 is finally measured with the qubit. The tunnelling probability $P_{\mathrm{t}}$ is plotted as a function of both the pump frequency and the delay $\Delta t_{r} . \mathbf{b}$, Fourier transform of the time-domain data. When starting with a single-photon Fock state, the frequency of the Ramsey oscillations is equal to the pump detuning $\Delta v_{\mathrm{p}}$.
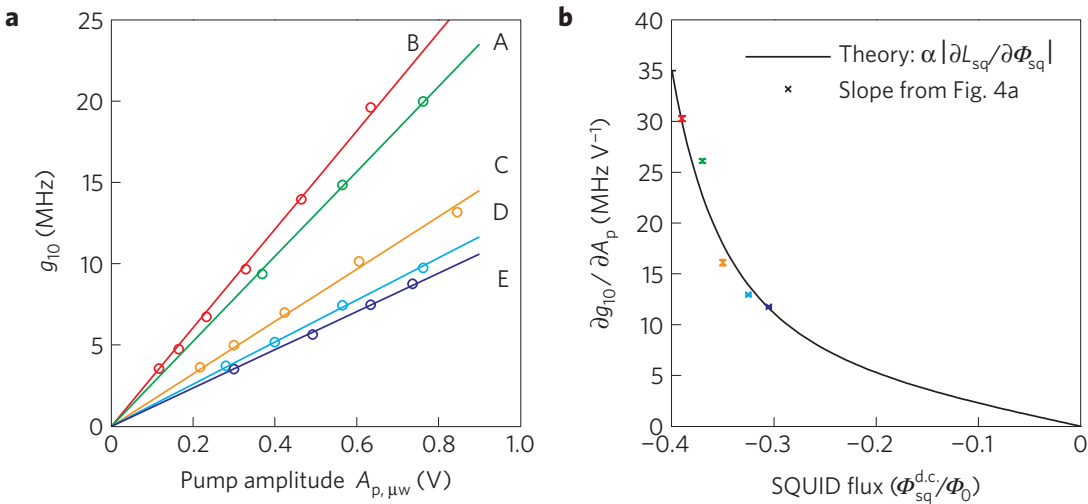

Figure 4 | Dependence of the coupling rate with the SQUID flux. a, Parametric coupling frequency $g_{10}$ between modes 0 and 1 as a function of the pump amplitude $A_{p, \mu w}$, for the various operating fluxes $A, B, C, D, E$ shown on Fig. 1c. In the explored flux and pump amplitude ranges, $g_{10}$ depends linearly on $A_{p, \mu w} \cdot \mathbf{b}$, Consistency check of the simple flux expansion of SQUID inductance used in our model (equation (1)). For a given flux, we extract the conversion efficiency $\partial g_{10} / \partial A_{p, \mu w}$ from the measurements in Fig. 4a. This is compared to a theoretical curve of $\alpha\left|\partial L_{J} / \partial \Phi\right|$, where $\left|\partial L_{J} / \partial \Phi\right|$ is the numerically calculated first derivative with flux of the SQUID inductance, and with $\alpha=\sqrt{\nu_{0} v_{1}} / 2 \sqrt{L_{0}^{\prime} L_{1}^{\prime}} M_{\mathrm{sq}} \kappa$, where $M_{\mathrm{sq}}$ is the mutual coupling inductance between the pump line and the SQUID, and $\kappa$ is the attenuation of the pump line. We set the value of the $\sqrt{\nu_{0} v_{1}} / 2 \sqrt{L_{0}^{\prime} L_{1}^{\prime}}$ prefactor to the one at point $A$, and we assume that $M_{\mathrm{sq}}$ is equal to the d.c. value $(1.2 \mathrm{pH}) . \kappa$ is the only adjustable parameter and is in good agreement, within $2 \mathrm{~dB}$, with the attenuation measured at room temperature.

microwave pulses and read out with a d.c. SQUID. By applying a fast flux pulse to the qubit, the $|\mathrm{e}\rangle$ state preferentially tunnels to a different flux state, which is indicated by a shift in the d.c. SQUID critical current. We measure a relaxation time $T_{1}^{\mathrm{q}} \approx 100 \mathrm{~ns}$, and a qubit-resonator coupling rate $g_{\mathrm{q} 1}=32 \mathrm{MHz}$. We load a single-photon Fock state in mode 1 by preparing the qubit in state $|e\rangle$ at $v_{\mathrm{q}}=10.116 \mathrm{GHz}$, bringing it in resonance with the cavity for a duration $T_{\pi}=1 / 4 g_{\mathrm{q} 1}=8 \mathrm{~ns}$, and shifting it back out of resonance. This prepares the cavity state $\left|0_{0} 1_{1}\right\rangle$ (the subscript denotes the mode number). After applying a pump pulse of duration $\Delta t_{\mathrm{p}}$, the state of mode 1 is transferred back to the qubit by bringing it again in resonance with the cavity for a duration $T_{\pi}$. Finally, we measure the state of the qubit. Figure $2 \mathrm{~d}(2 \mathrm{e})$ shows single photon conversion oscillations between modes 1 and 0 (resp. 1 and 2) as a function of $\Delta t_{\mathrm{p}}$ and $v_{\mathrm{p}}$. At zero pump detuning, the frequency of the oscillations is the lowest, equal to the splitting strength measured in spectroscopies 2 ( $\mathrm{a} \mathrm{b} \mathrm{c}$ ), and their amplitude is at their maxima. The
Fourier transforms of these data are computed in Fig. $2 \mathrm{f}$ and Fig. $2 \mathrm{~g}$ and exhibit the expected hyperbolic dependence $\sqrt{\left(2 g_{10}\right)^{2}+\Delta v_{p}^{2}}$ and $\sqrt{\left.\left(2 g_{21}\right)^{2}+\Delta v_{p}^{2}\right)}$, respectively, when the initial state is a singlephoton Fock state in one mode. The amplitude of these oscillations follows an exponential decay, with a characteristic time $T_{10}^{\text {Rabi }}$. Its measured value is $T_{10}^{\text {Rabi }} \approx 180 \mathrm{~ns}\left(T_{21}^{\text {Rabi }} \approx 100 \mathrm{~ns}\right)$, compatible with the harmonic mean of the measured relaxation times $T_{0}^{\text {Decay }} \approx 370 \mathrm{~ns}$ and $T_{1}^{\text {Decay }} \approx 140$ ns of both modes.

We measure the coherence of this parametric process by performing a Ramsey interference experiment with a photon 'shared' between modes 0 and 1 , as a function of the pump detuning, with a pump amplitude corresponding to $g_{10}=20 \mathrm{MHz}$. After preparing $\left|0_{0} 1_{1}\right\rangle$, we apply two phase-coherent pump pulses of $6 \mathrm{~ns}$ duration (corresponding to a $\pi / 2$ pulse at $\Delta v_{p}=0$ ), separated by a variable delay $\Delta t_{\mathrm{r}}$, and then measure the final state of mode 1. The first pulse prepares a superposition of states $\left|0_{0} 1_{1}\right\rangle$ and $\left|1_{0} 0_{1}\right\rangle$. During the free evolution, these two states acquire opposite 
phases differing by $2 \pi \Delta v_{\mathrm{p}} \Delta t_{\mathrm{r}}$. The second pulse combines these states, resulting in interference oscillations with a period equal to the inverse of the detuning. We verify this experimentally (Fig. 3). The amplitude of the oscillations decays on a characteristic timescale $T_{10}^{\text {Ramsey }} \approx 190 \mathrm{~ns}$, which indicates that the decoherence is mainly due to relaxation.

To further confirm the circuit model, we measure the $1 \leftrightarrow 0$ conversion rate (Fig. 4a) as a function of the pump amplitude, for different $\Phi_{\mathrm{sq}}^{\text {d.c. }}$ (points 'A, B, C, D, E' on Fig. 1c). For the flux bias range that we explore $\left([-0.4,-0.3] \Phi_{0}\right)$, the coupling rates $g_{10}$ are linearly dependent on the experimentally accessible pump amplitudes. The variation of the conversion efficiency $\partial g_{10} / \partial A_{\mathrm{p}, \mu \mathrm{w}}$ with the flux is also in good agreement with our SQUID inductor model and its simple expansion in equation (1), and is consistent with measured values of the pump line attenuation and d.c. mutual inductance to pump line (Fig. $4 \mathrm{~b}$ ).

To conclude, we have demonstrated that parametric frequency conversion is an effective way to coherently manipulate a single microwave-photon Fock state in frequency space. The dynamics are accurately described by a generalized beam-splitter interaction familiar from quantum optics. Combined with a phase shift operation, which can be implemented by fast shifts in SQUID bias flux, this system could be used as a novel linear optical quantum bit based on microwave resonator modes. Furthermore, straightforward technical improvements will enable the manipulation of multi-photon states. Finally, this device also offers the opportunity to explore other parametric interactions, such as amplification, with similarly strong interaction rates.

Received 30 March 2011; accepted 27 May 2011; published online 3 July 2011

\section{References}

1. Haroche, S. \& Raimond, J. Exploring the Quantum: Atoms, Cavities and Photons (Oxford Univ. Press, 2006).

2. Rauschenbeutel, A. et al. Controlled entanglement of two field modes in a cavity quantum electrodynamics experiment. Phys. Rev. A 64, 50301 (2001).

3. Wang, H. et al. Deterministic entanglement of photons in two superconducting microwave resonators. Phys. Rev. Lett. 106, 060401 (2011).

4. Brown, K. et al. Coupled quantized mechanical oscillators. Nature 471, 196-199 (2011).

5. Harlander, M., Lechner, R., Brownnutt, M., Blatt, R. \& Hänsel, W. Trapped-ion antennae for the transmission of quantum information. Nature 471, 200-203 (2011).

6. Louisell, W., Yariv, A. \& Siegman, A. Quantum fluctuations and noise in parametric processes. I. Phys. Rev. 124, 1646-1654 (1961).

7. Tucker, J. \& Walls, D. Quantum theory of parametric frequency conversion. Ann. Phys. 52, 1-15 (1969).

8. Wallraff, A. et al. Strong coupling of a single photon to a superconducting qubit using circuit quantum electrodynamics. Nature 431, 162-167 (2004)

9. Knill, E., Laflamme, R. \& Milburn, G. A scheme for efficient quantum computation with linear optics. Nature 409, 46-52 (2001).

10. Milburn, G. Photons as qubits. Phys. Scr. 2009, 014003 (2009).

11. Matthews, J., Politi, A., Stefanov, A. \& O’Brien, J. Manipulation of multiphoton entanglement in waveguide quantum circuits. Nature Photon. 3, 346-350 (2009).
12. Huang, J. \& Kumar, P. Observation of quantum frequency conversion. Phys. Rev. Lett. 68, 2153-2156 (1992).

13. Tanzilli, S. et al. A photonic quantum information interface. Nature 437, 116-120 (2005)

14. Rakher, M., Ma, L., Slattery, O., Tang, X. \& Srinivasan, K. Quantum transduction of telecommunications-band single photons from a quantum dot by frequency upconversion. Nature Photon. 4, 786-791 (2010).

15. Vandevender, A. \& Kwiat, P. High efficiency single photon detection via frequency up-conversion. J. Mod. Opt. 51, 1433-1445 (2004).

16. Gröblacher, S., Hammerer, K., Vanner, M. \& Aspelmeyer, M. Observation of strong coupling between a micromechanical resonator and an optical cavity field. Nature 460, 724-727 (2009).

17. Teufel, J. et al. Circuit cavity electromechanics in the strong-coupling regime. Nature 471, 204-208 (2011).

18. Wallquist, M. et al. Single-atom cavity QED and optomicromechanics. Phys. Rev. A 81, 23816 (2010).

19. Niskanen, A. et al. Quantum coherent tunable coupling of superconducting qubits. Science 316, 723 (2007).

20. Yurke, B. et al. Observation of parametric amplification and deamplification in a Josephson parametric amplifier. Phys. Rev. A 39, 2519-2533 (1989).

21. Bergeal, N. et al. Phase-preserving amplification near the quantum limit with a Josephson ring modulator. Nature 465, 64-68 (2010).

22. Castellanos-Beltran, M., Irwin, K., Hilton, G., Vale, L. \& Lehnert, K. Amplification and squeezing of quantum noise with a tunable Josephson metamaterial. Nature Phys. 4, 929-931 (2008).

23. Yamamoto, T. et al. Flux-driven Josephson parametric amplifier. Appl. Phys. Lett. 93, 042510 (2008).

24. Chirolli, L., Burkard, G., Kumar, S. \& DiVincenzo, D. P. Superconducting resonators as beam splitters for linear-optics quantum computation. Phys. Rev. Lett. 104, 230502 (2010).

25. Tian, L., Allman, M. \& Simmonds, R. Parametric coupling between macroscopic quantum resonators. New J. Phys. 10, 115001 (2008).

26. Hofheinz, M. et al. Synthesizing arbitrary quantum states in a superconducting resonator. Nature 459, 546-549 (2009).

27. Palacios-Laloy, A. et al. Tunable resonators for quantum circuits. J. Low Temp. Phys. 151, 1034-1042 (2008).

28. Sandberg, M. et al. Tuning the field in a microwave resonator faster than the photon lifetime. App. Phys. Lett. 92, 203501 (2008).

29. Louisell, W. Coupled Mode and Parametric Electronics (Wiley, 1960).

30. Sillanpää, M., Park, J. \& Simmonds, R. Coherent quantum state storage and transfer between two phase qubits via a resonant cavity. Nature 449, 438-442 (2007).

31. Law, C. K. \& Eberly, J. H. Arbitrary control of a quantum electromagnetic field. Phys. Rev. Lett. 76, 1055-1058 (1996).

\section{Acknowledgements}

We thank N. Bergren and L. Ranzani for technical help, and J. Park, F. Altomare and L. Spietz for valuable input.

\section{Author contributions}

E.Z-B. and F.N. designed the experiment, built the measurement set-up and performed the measurements. M.L., R.W.S., J.A. contributed to the experimental design. L.R.V. contributed to the fabrication process development. J.A. conceived the experiment and supervised the project. All authors participated in the sample fabrication, the writing of the manuscript and the data analysis.

\section{Additional information}

The authors declare no competing financial interests. Supplementary information accompanies this paper on www.nature.com/naturephysics. Reprints and permissions information is available online at http://www.nature.com/reprints. Correspondence and requests for materials should be addressed to E.Z-B, F.N. or J.A. 